\title{
Gamma-Ray Dose Measurement with Radio-Photoluminescence Glass Dosimeter in Mixed Radiation Field for BNCT
}

\author{
K. Hiramatsu ${ }^{1}$, S. Yoshihashi ${ }^{2}$, S. Kusaka ${ }^{1}$, F. Sato $^{1}$, E. Hoashi ${ }^{1}$, I. Murata $^{1}$ \\ ${ }^{1}$ Graduate School of Engineering, Osaka University, Yamada-oka 2-1, Suita, Osaka 565-0871, Japan \\ ${ }^{2}$ Department of Applied Nuclear Technology, Fukui University of Technology, Gakuen 3-6-1, Fukui-city, Fukui 910-8505, Japan
}

\begin{abstract}
Accelerator based neutron sources (ABNS) are being developed as the next generation neutron irradiation system for BNCT. From the ABNS, unnecessary gamma-rays will be generated by neutron capture reactions, as well as fast neutrons. To control the whole-body radiation dose to the patient, measurement of gamma-ray dose in the irradiation room is necessary. In this study, the objective is to establish a method to measure gamma-ray dose separately in a neutron/gamma mixed field by using RPL glass dosimeter. For this purpose, we proposed a lead filter method which uses a pair of RPL glasses with and without a lead filter outside. In order to realize this method, the basic characteristics of glass dosimeter was verified in the gamma-ray field, before adapting it in the mixture field. From the result of the experiment using the lead filter, the simulation result especially for the case with a lead filter overestimated the absorbed does obtained from measurement. We concluded that the reason of the discrepancy is caused by existence of gradient of the dose distribution in the glass, and the difference of sensitivity to low-energy photon between measurement and theory.
\end{abstract}

\section{Introduction}

The number of people who die of cancer is increasing all over the world. Especially in Japan, according to the cause-specific ranking of death cancer has become first place since 1981 [1]. Furthermore, as aging of population progresses, the number of cancer morbidity and/or death is expected to increase in the future. Therefore, in modern medical science, development of cancer therapies is an urgent task. Mainly, cancer treatment can be classified into surgical therapy, chemotherapy and radiotherapy. There are some advantages and disadvantages in each treatment, and the optimal treatment method of individual has thus been selected by considering each case of cancer. Further, it is possible to enhance the therapeutic effect by combining these treatment methods.

Recently, radiotherapy has attracted attention with development of accelerator technology. In radiotherapy, cancer cells will be destroyed by ionization effect of radiation, which injure the DNA in living cells. Because it is possible to irradiate around the cancer cells, radiation therapy can be regarded as a local treatment. Since forms or functions of organs will be preserved in radiotherapy, invasion to the normal tissues can be suppressed and quality of life can consequently be maintained fairly well after treatment compared with surgical therapy. In addition, general anesthesia of surgery or chemotherapy with an anti-cancer agent will become a heavy burden to a human body, especially for elderly patients, while it is possible to perform radiotherapy almost regardless of age of the patients because radiotherapy is a minimally invasive treatment. Therefore, radiotherapy is expected to be a treatment that can maintain QOL at high levels.

Ideally, as for radiotherapy, only target cancer cells will be irradiated, while normal cells surrounding the target will not be irradiated at all. In fact, however, since the target cells are irradiated from outside of the body, not only cancer cells but also normal cells will possibly be affected by radiation. Generally, radiation damage of cancer cells is almost the same as that of normal cells, while it takes more time to recover from the damage for cancer cells compared to normal cells. Therefore, actual treatments are performed with a special devise so as to destroy only cancer cells while suppressing the damage to normal cells, e.g., irradiation of the target is repeatedly carried out with a certain time interval for which normal cells will recover from the damage.

Radiation therapy is divided into two categories for the type of incident radiation; indirectly ionizing radiation such as X-rays and gamma rays, and directly ionizing radiation such as particle beam which normally uses heavier particles than electrons. In the former, the dose is at maximum on the surface of the body and is attenuating with increase of the depth in the body, so that the therapeutic effect can hardly be obtained if cancer cells are in a deep position inside the body. Furthermore, if we try to give a sufficient dose to cancer cells located deep inside the body, it would significantly affect the normal cells shallower than the cancer cells. Therefore, in a cyber knife and gamma knife using X-rays and 
gamma rays, respectively, cancer cells are irradiated with relatively weak dose radiation from multiple directions. As the result, only cancer cells will be irradiated with a sufficient dose, while the radiation effects on the normal cells can be suppressed.

On the other hand, as for the particle beam therapy, the pass length of incident charged particles is determined by the incidence energy, and the particles transfer a very large energy to surrounding cells, when stopping after losing the whole energy (Bragg peak). Therefore, the Bragg peak can be adjusted to match the cancer cell depth by changing extracted energy from the accelerator. For that reason, irradiation effects on normal cells existing between the body surface and cancer cells can be minimized. At the actual treatment, the Bragg peak is expanded with various energetic particles to cover the whole cancer cells (Spread Out Bragg Peak ; SOBP).

Recently, a new radiotherapy, BNCT (Boron Neutron Capture Therapy), is known as a quite new cancer treatment by which damage to normal cells can be substantially smaller than other radiation therapies. Since boron has a specific property of easily reacting with neutrons, cell selective radiotherapy is realized.

\subsection{Boron Neutron Capture Therapy}

BNCT is one of the particle beam therapies which uses a nuclear reaction of boron and neutron. Equation.a indicates the reaction of boron (B) and neutron [2].

$$
{ }^{10} \mathrm{~B}+\mathrm{n} \rightarrow\left\{\begin{array}{c}
{ }^{4} \mathrm{He}+{ }^{7} \mathrm{Li}(2.79 \mathrm{MeV}) \cdots 6 \% \\
{ }^{4} \mathrm{He}+{ }^{7} \mathrm{Li}(2.31 \mathrm{MeV})+\gamma(0.48 \mathrm{MeV}) \cdots 94 \%
\end{array}\right.
$$

Table 1-1 shows the reaction cross-sections of thermal neutrons for boron-10 and other elements constituting the human body. From the table, it is seen that boron-10 has a large reaction cross-section for thermal neutrons compared with the other elements. Figure 1-1 shows a schematic diagram of BNCT.

In this radiotherapy, high concentration of boron drug; $20 \mu \mathrm{g} / \mathrm{g}$, will be accumulated in cancer cells before irradiation of neutrons from outside of the body. After the irradiation, alpha particles and lithium nuclei generated in the reaction of Eq. (1), will destroy cancer cells. Since alpha particles and Li-7 nuclei have very short range of about 4-9 $\mu \mathrm{m}$, this radiotherapy can destroy only cancer cells selectively without affecting normal cells around the cancer cells.

Table 1-1. Reaction cross-sections of thermal neutrons for some materials.

\begin{tabular}{l|l|l} 
Nuclide & Reaction & Cross section [barn] \\
\hline${ }^{1} \mathrm{H}$ & ${ }^{1} \mathrm{H}(\mathrm{n}, \gamma)^{2} \mathrm{H}$ & 0.332 \\
${ }^{12} \mathrm{C}$ & ${ }^{12} \mathrm{C}(\mathrm{n}, \gamma){ }^{13} \mathrm{C}$ & 0.0035 \\
${ }^{14} \mathrm{~N}$ & ${ }^{14} \mathrm{~N}(\mathrm{n}, \mathrm{p}){ }^{14} \mathrm{C}$ & 1.83 \\
${ }^{16} \mathrm{O}$ & ${ }^{16} \mathrm{O}(\mathrm{n}, \gamma){ }^{17} \mathrm{O}$ & 0.00019 \\
${ }^{10} \mathrm{~B}$ & $\left.{ }^{10} \mathrm{~B}(\mathrm{n}, \alpha)\right)^{7} \mathrm{Li}$ & 3838
\end{tabular}



Fig 1-1. Schematic diagram of BNCT.

For advances in the treatment technology of BNCT, further investigation is required in several fields, i.e., drug delivery techniques that can administer boron to cancer cells, development of the neutron source instead of nuclear reactors and radiation dosimetry at the time of treatment. In particular, the development of the neutron source is an essential issue for the development of BNCT. Because BNCT requires a high flux neutron field, treatment has been performed only with research reactor until now. However, there are several problems for reactor based BNCT. At first, the number of research reactors that can perform neutron irradiation is not so many. As a result, patients are required to move to the nuclear reactor consuming a long time. It is a severe burden on the patient's body. Further, since the regulation of the nuclear reactor is very strict and hard, it is prohibited to build inside or adjacent to hospitals from the standpoint of safety. Therefore, accelerator based neutron sources (ABNS) are being developed as the next generation irradiation system for BNCT instead of nuclear reactors.

ABNS is a facility that can irradiate neutrons generated by nuclear reaction of accelerated particles and target materials. The neutron beam characteristics are controlled by a so-called beam shaping assembly containing neutron moderator and radiation shields. ABNS can be designed compactly compared with the nuclear reactor, and its inherent safety enables to install an ABNS in a hospital. However, for commercial use of the ABNS, further investigation is necessary such as accelerator, target and beam shaping assembly developments.

At present, liquid lithium, solid lithium and solid beryllium have been considered as the target materials of ABNS. Each material has advantage and disadvantage. In Osaka University, development of liquid lithium target ABNS is underway.

\section{2 Objectives}

In the ABNS, neutrons generated by the reaction of protons and target are moderated in the beam shaping assembly. In the process, unnecessary gamma-rays are produced by neutron capture reaction of neutrons with materials arranged on the transport route to the beam exit. In radiology, it is very important to reduce impact on normal cells by secondary radiation as much as possible. It can affect the whole body exposure dose of patient. Because development of ABNS has been just started, the characteristics of the secondary radiations in the 
irradiation field of the ABNS such as neutrons and gamma-rays can change depending on the type of accelerators and thus have not been understood. Therefore, measurement of gamma-ray dose given at the same time is necessary for controlling the whole body radiation dose of patients.

There are several detectors which are used for measurement of gamma-rays. For example, GeigerMüller counter, ionization chamber, scintillation detector, semiconductor detector, thermoluminescent dosimeter (TLD), radio-photoluminescence glass dosimeter (RPLDG) etc. are known so far. Among these detectors, most of detectors can measure time dependent dose rate of gamma-rays. On the other hand, TLD and RPLGD are used for measuring the cumulative dose at the measurement position. Until now, TLD is widely used for dosimetric applications to determine absorbed dose in radiotherapy. TLD has good reproducibility and small energy dependence [3]. However, it is quite difficult to handle because it has fading characteristics and its dose information will be deleted by dose measurement. Thus, RPLGD has recently been used for dosimetry instead of TLD. There is almost no fading characteristics in RPLGD, and it can be used easily.

Generally, for the dosimetry in the field where neutrons and gamma-rays exist, it is known that it is difficult to measure each radiation dose separately, because the detectors have sensitivity to both of radiations. This is also the case of using a RPLGD. Normally, RPLGD is used for environmental monitoring at nuclear reactor facility and its surrounding. Therefore, it is not designed to apply in the mixed field of neutron and gamma-ray. In other words, for the measurement in a strong neutron field, since gamma-rays are also existing, it is very difficult to measure gamma-ray dose separately. Until now, several detectors are proposed for measurement in the mixed field of neutrons and gamma-rays such as TLD and ionization chamber. However, these detectors still have problems. TLD has fading characteristics and it is impossible to be readout repeatedly. Ionization chamber also has some problems such as the size and difficulty of handling. In BNCT project of Osaka University, RPLDG is adopted for measurement of gamma-rays in the irradiation room from the view point of its size and ease of use. Therefore, the aim of this study is to establish a new measurement method with RPLDG which can evaluate only gamma-ray dose from dose values measured in a mixed field of neutrons and gamma-rays in BNCT.

To evaluate the gamma-ray dose from the measured dose, we have devised a new measurement method which uses a lead shield. The key is that neutrons can more-or-less penetrate the lead shield, on the other hand, gamma-rays will be attenuated partly by the lead. We call it "A lead filter method."

In this method, bare RPLGD and RPLGD covered with a lead filter were irradiated at the same irradiation field. By making difference of both measured values, the effect of neutrons will be cancelled, and only the gamma-ray dose can be shielded by the lead shield and a part of the dose will remain. The real gamma-ray dose can be estimated from the measured dose of RPLGD and the correction coefficient evaluated beforehand.

In the present study, since the reaction of neutrons and gamma-rays differ with the elements, correction coefficient may vary depending on the energy variation. In this study, to determine the correction coefficient used for this method, we evaluate the energy dependence of the measurement by RPLGD covered by a lead filter in the gamma-ray field as the first step of this method before adapting in mixed field.

\section{Materials and Methods}

\subsection{RPLGD}

RPLGD is one of the integrated-type individual dosimeters which is currently put into practical use. RPLGD is composed of silver activated phosphate glass. The glass irradiated with radiation emits orange light by stimulation of ultraviolet beam. This phenomenon is referred to as radio-photoluminescence (RPL). By utilizing the characteristics, that is, the amount of light emission is proportional to exposed dose, RPLGDs are mainly used for measurement of gamma-ray dose. Figure 2-1 shows a schematic diagram of the generation mechanism of an RPL center in a silver activated phosphate glass. As an initial condition, a silver (Ag) atom contained in the silver activated phosphate glass is stable in the state of $\mathrm{Ag}^{+}$. After irradiation, a pair of electron and hole is generated inside the glass. The generated electron is trapped in $\mathrm{Ag}^{+}$to create $\mathrm{Ag}^{0}$. On the other hand, the generated hole is once captured by a $\mathrm{PO}_{4}$ tetrahedron, and after that, the hole charge is shifted to $\mathrm{Ag}^{+}$to finally make a stable $\mathrm{Ag}^{2+}$. This phenomenon is called RPL center build-up [4]. Figure 2-2 indicates the schematic diagram of RPL emission process of silver active phosphate glass due to ultraviolet excitation.

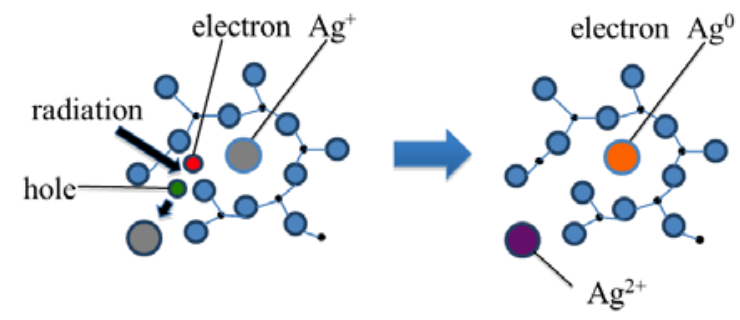

Fig 2-1. Schematic diagram of the generation mechanism of an RPL center.

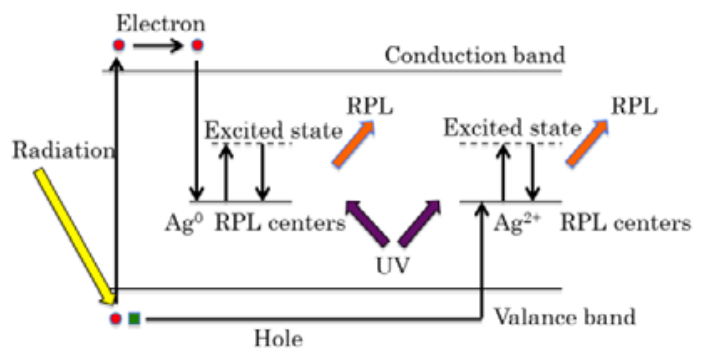

Fig 2-2. Schematic diagram of RPL emission process. 
The RPLGD measurement system (Dose Ace, AGC Asahi Glass Company, Japan) is composed of RPLGD (GD-301, AGC Asahi Glass Company) and a readout system (Dose Ace FGD-1000, AGC Asahi Glass Company). Figure 2-3 is a photo of RPLGD. As shown in this figure GD-301 is $1.5 \mathrm{~mm}$ in diameter and $8.5 \mathrm{~mm}$ in length. The weight composition is $\mathrm{P}(31.55 \%), \mathrm{O}$ (51.16\%), $\mathrm{Al}(6.12 \%), \mathrm{Na}(11.0 \%)$ and $\mathrm{Ag}(0.17 \%)$, and the density is 2.61 [5]. For the readout, ultraviolet excitation light source is required to induce RPL. As for the ultraviolet source, LD-pumped ultraviolet solid-state laser (Explorer 349, Spectra-Physics) is implemented in FGD-1000. The laser is collimated in $1 \mathrm{~mm}$ in diamiter through an aperture. The amount of RPL measured by the readout device is calibrated by that of a refference RPLGD which was irradiated beforehand and already known the accurate absorbed dose.

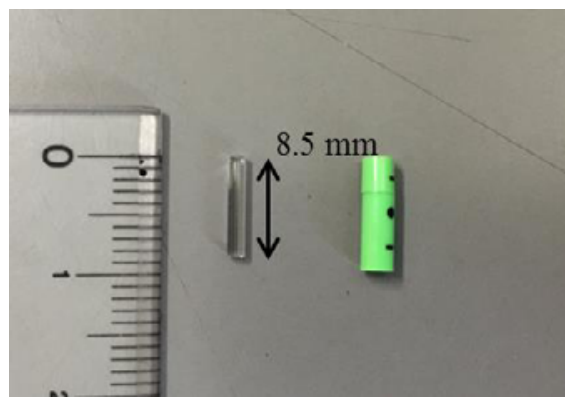

Fig 2-3. Photo of RPLGD.

\subsection{Lead Filter Method}

In this study, we aimed to establish a new measurement method; "lead filter method", to realize a separate measurement of gamma-ray dose in a mixed field of neutrons and gamma-rays. This method utilizes the characteristics that lead transmits neutrons, on the other hand, shields gamma-rays if the thickness of the lead is appropriately thin. Under this assumption, the measured value of absorbed dose D of the bare RPLGD in the mixed field can be derived by the following equation.

$$
\mathrm{D}=\mathrm{d} \gamma+\mathrm{dn}+\mathrm{dn} \gamma
$$

On the other hand, the measured value of absorbed dose $\mathrm{D}_{\mathrm{Pb}}$ of the RPLGD covered with a lead filter can be derived by the following equation.

$$
\mathrm{D}_{\mathrm{Pb}}=\mathrm{d}^{*} \gamma+\mathrm{dn}+\mathrm{dn} \gamma
$$

Here, $d n$ and $d \gamma$ are the dose of neutrons and gammarays, respectively. $\operatorname{dn} \gamma$ is the dose of gamma-rays induced by neutron capture reaction. As the reaction of gamma-ray, photonuclear reaction; $(\gamma, n)$ is also considered. However, since this nuclear reaction will not occur unless the incident photon energy is higher than the threshold energy of each element, this nuclear reaction can be ignored in this method. Further, $d^{*} \gamma$ is dose of gamma-ray attenuated by the lead filter, and can be written as equation (3) with transmittance factor: $\varepsilon$.

$$
\mathrm{d}^{*} \gamma \fallingdotseq \varepsilon \mathrm{d} \gamma
$$

By making difference of the two measured values, the neutron effect can be removed, as shown in equation (4).

$$
\begin{aligned}
\mathrm{D}-\mathrm{D}_{\mathrm{Pb}} & =\mathrm{d} \gamma-\mathrm{d}^{*} \gamma \\
& =(1-\varepsilon) \mathrm{d} \gamma
\end{aligned}
$$

Finally, the absorbed dosed which we want to know can be deduced with a correction factor by equation (5).

$$
\mathrm{d} \gamma=\left(\mathrm{D}-\mathrm{D}_{\mathrm{Pb}}\right) /(1-\varepsilon)
$$

The gamma-ray dose can be estimated by correcting the measured values of bare and lead filtered RPLGDs with the correction factor estimated in advance.

Figure 2-4 shows the lead filter used in this study. It is a cylinder the size of which is $2.2 \mathrm{~cm}$ in diameter and 8.5 $\mathrm{cm}$ in length. The thickness of the lead is designed as 1 $\mathrm{cm}$, which can shield gamma-rays partly, on the other hand, will not affect the neutron dose.

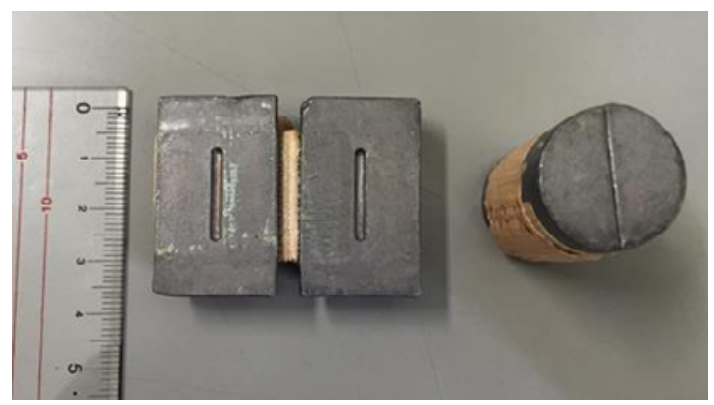

Fig2-4. Lead filter used in this study.

\subsection{Simulation Method}

In order to analyse the presently carried out experimental results PHITS (Particle Heavy Ion Transport code System) was used. PHITS is a general purpose Monte Carlo particle transport simulation code developed in Japan, in which EGS5 (Electron Gamma Shower 5) is implemented for precise gamma-ray and electron transport. As for neutron reaction cross section library, JENDL-4.0 was used. In the simulation, the experimental system was modelled precisely [6].

\subsection{Experimental Method}

It is conceivable that dosimetry using RPLGDs with a lead filter depends on various factors, such as kind of radiation and its energy. In fact, when this method is applied to treatment field of BNCT, it is a mixed field of mainly gamma-rays and neutrons. Therefore, it is essential to evaluate the energy dependence of RPLGD in a gamma-ray field, neutron field and mixed field of gamma-ray and neutron. In this study, we evaluate the energy dependence of RPLGD in the gamma-ray field as the first step of development of this method. To realize this, irradiation experiments with standard gamma-ray sources have been carried out. 
As for standard gamma-ray sources, ${ }^{60} \mathrm{Co},{ }^{137} \mathrm{Cs}$ and ${ }^{22} \mathrm{Na}$ were used. Table 2-1 summarizes the details of the sources. Figure 2-5 shows the size and photo of the standard gamma-ray source. This type of a radiation source is a point source the size of which is normally smaller than $6 \mathrm{~mm}$ in diameter. Aluminium foil of about $0.1 \mathrm{~mm}$ thick and acrylic resin are used for the source window and casing, respectively. In the analysis of experiments, the structure of the sources was accurately modelled.

Table 2-1. Details of the sources.

\begin{tabular}{l|l|l|l|l} 
Nuclide & $\begin{array}{l}\text { Intensity } \\
{[\mathrm{kBq}]}\end{array}$ & $\begin{array}{l}\text { Half } \\
\text { Life } \\
\text { time } \\
{[\text { year }]}\end{array}$ & $\begin{array}{l}\text { Energy } \\
{[\mathrm{MeV}]}\end{array}$ & $\begin{array}{l}\text { Emission } \\
\text { Ratio[\%] }\end{array}$ \\
\hline \hline${ }^{60} \mathrm{Co}$ & 1060 & 5.27 & 1.173 & 100 \\
\cline { 4 - 5 } & & & 1.333 & 100 \\
\hline${ }^{137} \mathrm{Cs}$ & 1000 & 30.17 & 0.662 & 85.1 \\
\hline${ }^{22} \mathrm{Na}$ & 867 & 2.60 & 1.275 & 100 \\
\cline { 4 - 5 } & & & 0.511 an. & 180
\end{tabular}

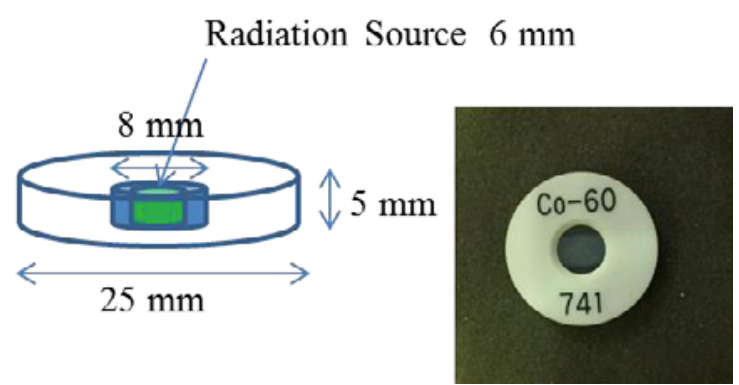

Figure 2-5. Size and photo of the standard gamma-ray source.

Figure 2-6 shows the experimental setup. The experimental setup was made on an aluminium plate of which the thickness is $5 \mathrm{~mm}$. Both RPLGDs with and without a lead filter were put on $5 \mathrm{~cm}$ from the radiation source and an irradiation experiment was performed. Irradiating three pairs of both RPLGDs, the averaged values were measured. RPLGDs without a lead filter were put on a styrofoam foundation in order to fix the measurement position. Further, measurement results were compared with calculation results which simulate the experimental system exactly. The background dose in the irradiation room was measured by Geiger Muller survey meter showing that the air dose rate was less than $1 \mu \mathrm{Sv} / \mathrm{hr}$.

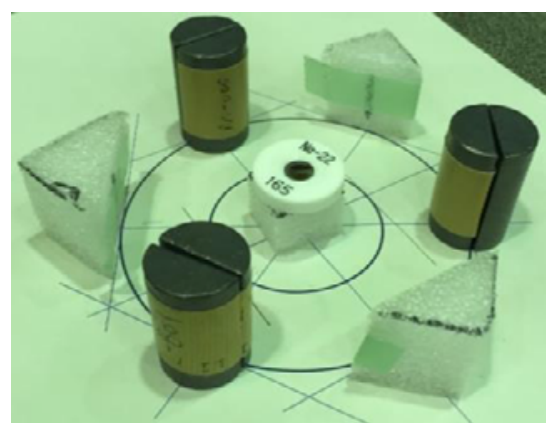

Figure 2-6. Experimental setup.

\section{Result}

We performed irradiation experiments with standard gamma-ray sources listed in Table 2-1. Figure 3-1 shows the comparison results of absorbed dose of RPLGD between measurement and simulation for each radiation source. Figures 3-1.a and 3-1.b are the results with and without lead filters, respectively. From the results, in the case of the RPLGD without a lead filter, measurement and simulation result agree well except ${ }^{60} \mathrm{Co}$ source. For the ${ }^{60} \mathrm{Co}$ source, the simulation value becomes smaller than the measurement value. In contrast, the simulation values show a little larger than the measurement for the RPLGDs with lead filters for all radiation sources.



Fig 3-1.a. Comparison result of absorbed dose between measurement and simulation for bare RPLGDs.

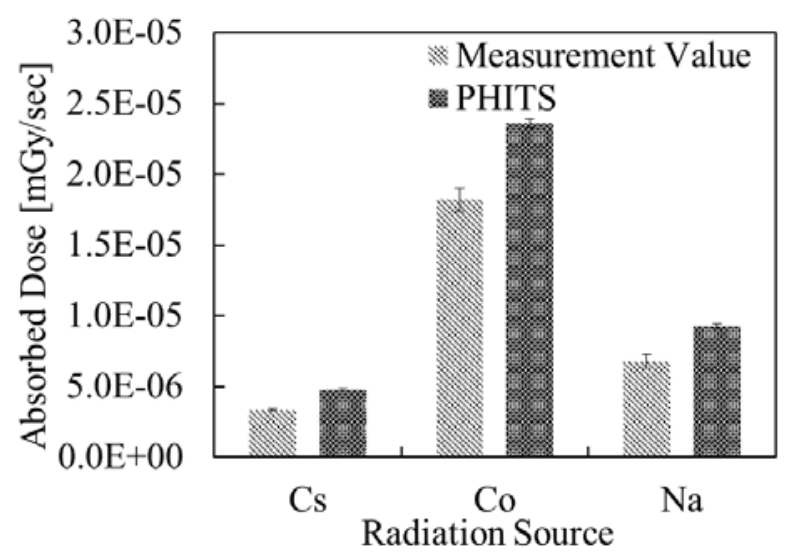

Fig 3-1.b. Comparison result of absorbed dose between measurement and simulation. RPLGDs were covered with a lead filter.

To clarify the discrepancy, we focused on two problems, dose distribution inside a glass and sensitivity to lowenergy photon. As for the first problem, it should be noted that the readout volume of the glass is fixed to be 6 $\mathrm{mm}$ in length and $1 \mathrm{~mm}$ in diameter inside the glass due to the technical restriction of the reader and the diameter of ultraviolet beam. Figure 3-2 shows the size of readout volume inside the RPLGD. In the measurement, the estimated absorbed dose is the averaged value only inside the readout volume. It means, if the dose 
distribution inside a glass is not uniform, absorbed doses of the entire glass and only of the readout volume will not agree. For accurate measurement, it is necessary to evaluate distribution of absorbed dose inside a glass. The details of the dose distribution inside a glass are discussed a little later in Sec. 3.1.

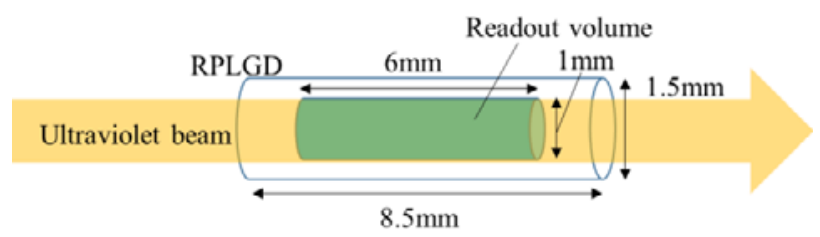

Fig 3-2. Size of readout volume inside RPLGD.

For another reason for the discrepancy of measurement and simulation, the sensitivity of RPLGD for radiation, especially for low-energy photon was examined. When high-energy gamma-rays are incident to lead, lowenergy characteristic X-rays will be generated. Figure 33 shows the photon flux calculated by PHITS, which was incident on the glass from the lead filter. The source was ${ }^{137} \mathrm{Cs}(662 \mathrm{keV})$. From this figure, it was found that many low-energy photons can be generated by interaction of gamma-rays and lead atoms.

It is known that RPLGD has high sensitivity to lowenergy photons [7]. Fig 3-4 shows the theoritical value of the mass energy absorption coefficient ratio of the air to the glass calculated from the composition ratio of the glass elements [8]. From the figure, it is found that RPLGD has high sensitivity to photon of which the energy is less than $100 \mathrm{keV}$. Since in this energy region, a lot of characteristic X-rays are produced as shown in Fig. 3-3, we presumed that there would be discrepancy of sensitivity to photons between measurement and simulation. In order to comfirm this, we performed experiments to examine whether the theoritical value of the mass energy absorption coefficient ratio calculated by PHITS is consistent with the dose response of actual RPLGD. The detailed results will be discussed in Sec. 3.2 .

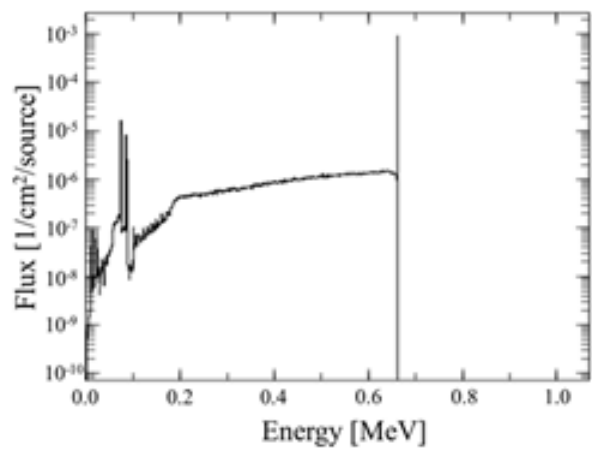

Fig 3-3. Calculated photon spectrum in the glass with a lead filter. Simulation was performed with ${ }^{137} \mathrm{Cs}$ source.

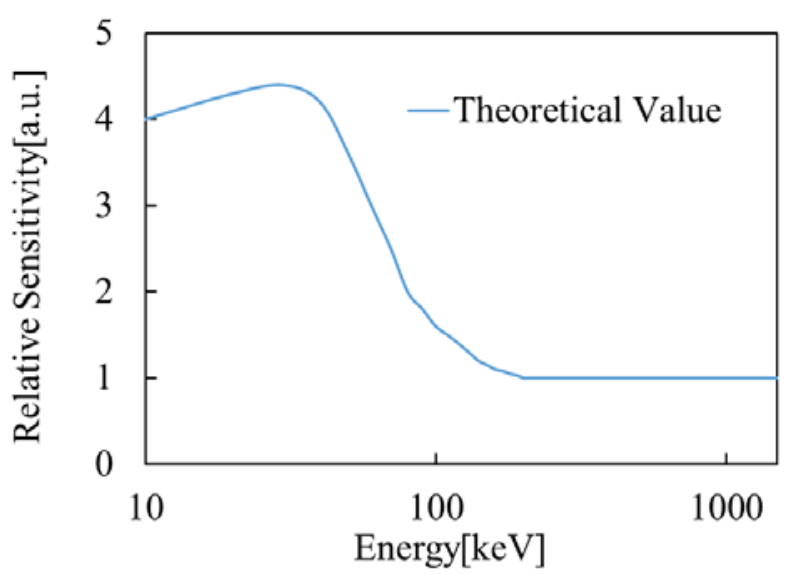

Fig.3-4. Theoritical value of the mass energy absorption coefficient ratio of the air to glass calculated from the composition ratio of the glass element.

\subsection{Dose Distribution}

In this section, the absorbed dose distribution in the glass is discussed. In the next section, the sensitivity examination to low energy photons is detailed based on the experimenatl results. To evaluate distribution of absorbed dose inside a glass, cross section of the glass was subdivied, and absorbed dose of each voxel was calculated by PHITS for the experimental setup of Figure 2-6. Figure 3-5 shows the calculation model of the cross section of a glass. Shaded area indicates the readout volume of RPLGD. The size of each voxel is $0.075 \times 0.075 \times 8.5 \mathrm{~mm}^{3}$. The distribution of absorbed dose was calculated in the parallel and vertical directions with respect to the gamma-ray inicidence direction as indicated in red frame. By simulating ${ }^{60} \mathrm{Co}$ and ${ }^{137} \mathrm{Cs}$ gamma-rays as a radiation source, energy dependent dose distribution can be discussed.



Fig 3-5. Calculation model of cross section of a glass.

Figures 3-6 and 3-7 show the simulation results of dose distribution for parallel and vertical directions inside a glass for ${ }^{137} \mathrm{Cs}$ and ${ }^{60} \mathrm{Co}$ gamma-ray sources, respectively. Shaded areas on the graph indicate the readout volume of RPLGDs. As shown in the figures, in the case for RPLGDs without a lead filter, the absorbed doses near the center of the glass are higer than those of around the peripheral region. On the other hand, in the case for 
RPLGDs with a lead filter, absorbed doses around the periphery become higher compared with the doses near the center of the glass. Furthermore, as focusing on the parallel direction, the shape of the curve of dose distribution differs depending on the energy $\left({ }^{137} \mathrm{Cs}\right.$ and ${ }^{60} \mathrm{Co}$ ), in the case for RPLGDs with a lead filter. As for ${ }^{137} \mathrm{Cs}$, the absorbed dose of the nearest position to the radiation source was the highest. On the other hand, in the case of ${ }^{60} \mathrm{Co}$, the deeper the position is from the radiation source, the more the absorbed dose increases. In the measurement, the real absorbed dose is the averaged value only inside the readout volume indicated as the shaded part in the graph. It means absorbed dose in the peripheral region of RPLGDs were removed in the measured absorbed doses. This is the reason why descrepancy is observed between measurement and simulation.

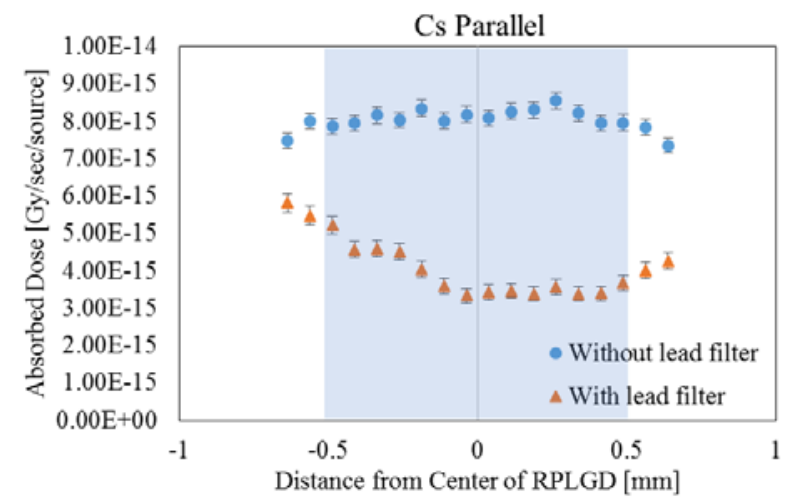

Fig 3-6.a. Simulation results of dose distribution of parallel direction. ${ }^{137} \mathrm{Cs}$ was simulated as a radiation source.



Fig.3-6.b Simulation results of dose distribution of vertical direction. ${ }^{137} \mathrm{Cs}$ was simulated as a radiation source.

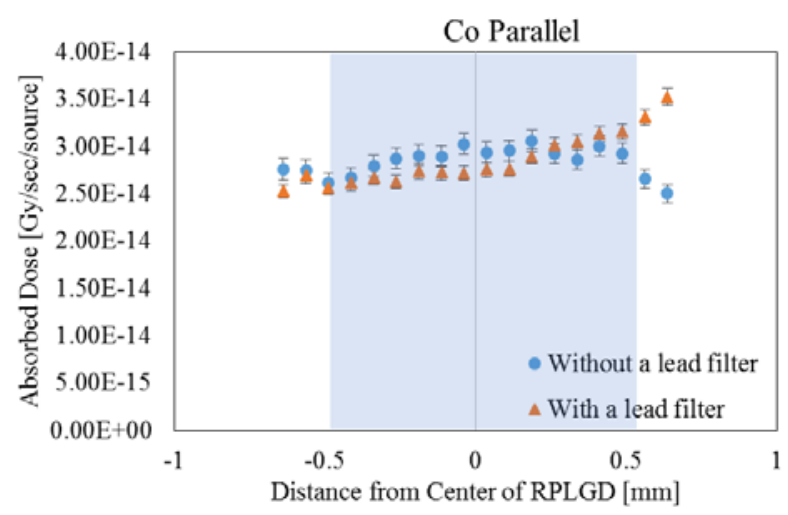

Fig 3-7.a. Simulation results of dose distribution of parallel direction. ${ }^{60} \mathrm{Co}$ was simulated as a radiation source.



Fig.3-7.b Simulation results of dose distribution of vertical direction. ${ }^{60} \mathrm{Co}$ was simulated as a radiation source.

\subsection{Dose Sensitivity}

We carried out irradiation experiments using low-energy X-rays to compare the theoretical sensitivity of RPLGDs to photons with the actual dose sensitivity of the glass. From the results in the previous experiments using gamma-ray source and RPLGDs without a lead filter, measurement and simulation agreed fairly well. We thus focused on the sensitivity especially for low-energy photons of which energy is less than $100 \mathrm{keV}$, comparable to energy of characteristic X-rays generated by interaction of gamma-rays and lead atoms. Figures 38.a and 3-8.b are the photos of an X-ray irradiation system (MBR-1520R-4, Hitachi Power Solutions) and the experimental arrangement of irradiation, respectively. By measuring the spatial dose rate at the irradiation position beforehand, it is possible to adjust the actual irradiation dose accurately. Table 3-1 shows the irradiation condition. Irradiating three pairs of RPLGDs at the same time, the obtained values were averaged to be the measured value. Dose sensitivity of RPLGD was calculated by making the ratio of irradiation dose and measured absorbed dose.

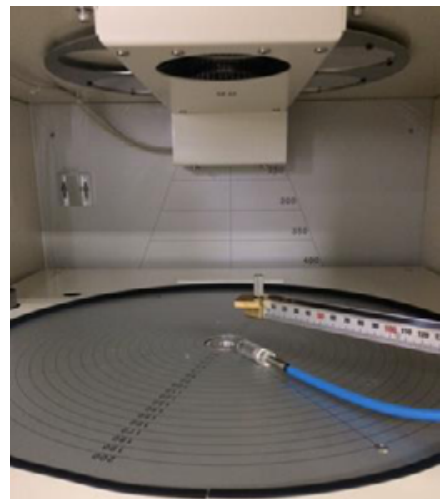

Fig 3-8.a. Photo inside the X-ray irradiation system. 


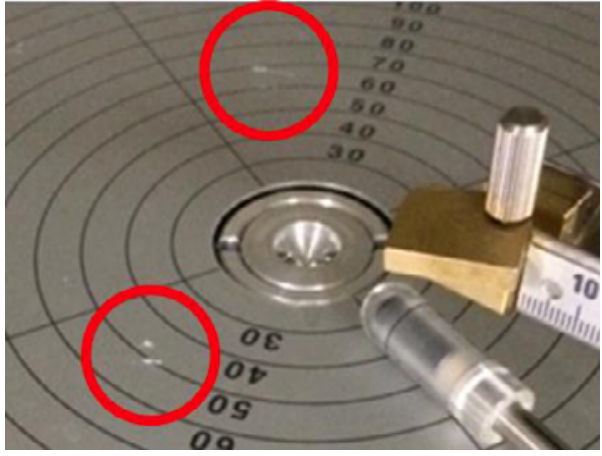

Fig 3-8.b. Photo of irradiation situation.

Table 3-1. Irradiation condiation.

\begin{tabular}{l|l} 
Tube Current & $10 \mathrm{~mA}$ \\
\hline Tube Voltage & $150 \mathrm{kV}$ \\
\hline Energy & $10,25,39,45,55,65 \mathrm{keV}$ \\
\hline Irradiation Dose & $100 \mathrm{mGy}$
\end{tabular}

Figure 3-9 shows a comparison result of sensitivity to photon between measurement and theoretical value calculated by mass energy absorption coefficient. As the result, the measured sensitivity tends to become lower than the theoretical value. Since many characteristics Xrays will be produced if using the lead filter, we concluded that the reason of the disagreement between experimental values and simulation in Figure 3-1.b has been caused by the decline of the sensitivity to the lowenergy photons in measurement.

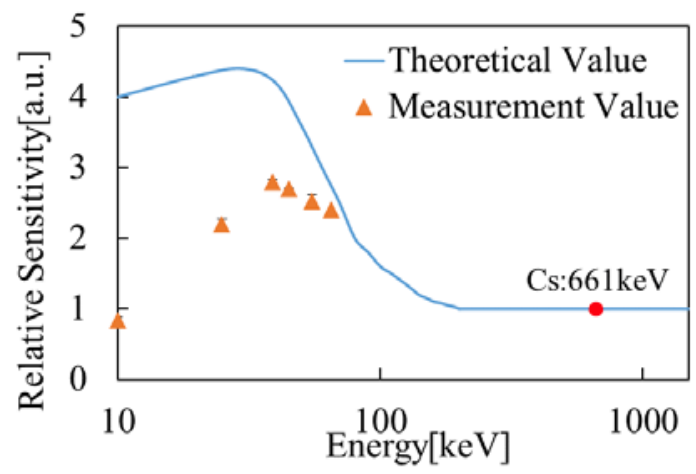

Fig 3-9. Comparison result of sensitivity to photon between measurement and theoretical value.

\subsection{Recalculation Result}

Based on the results above, absorbed dose of glass dosimeter was recalculated taking into account the dose distribution in a glass and the sensitivity for low-energy photons. In the recalculation, tally region in the simulation model was set to fit to the actual readout volume in the glass. Figure 3-10 shows the results of recalculation. From the figure, in both cases with/without a lead filter, the difference of values between measurement and simulation is reduced by the correction. In detail, for RPLGD without a lead filter the simulation value increases. On the other hand, for RPLGDs with a lead filter, the simulation value decreases by the correction. However, gamma-ray dose could not be reduced sufficiently even if using a lead filter in case of ${ }^{60} \mathrm{Co}$. It means, if the energy is too large, the strength of gamma-rays may not be lowered enough by the lead filter. In other words, the lead filter method may not be utilized if the gamma-ray dose could not be attenuated sufficiently with a lead filter.

By the current structure of the filter, this method can be applied to gamma-rays the energy of which is about $1 \mathrm{MeV}$ or less. At the treatment field of BNCT higher energy gamma-rays than ${ }^{60} \mathrm{Co}$, up to about $10 \mathrm{MeV}$, will be produced by neutron capture process. We thus need to re-examine gamma-ray attenuation process, i.e., the structures of the lead filter and glass in the next step.

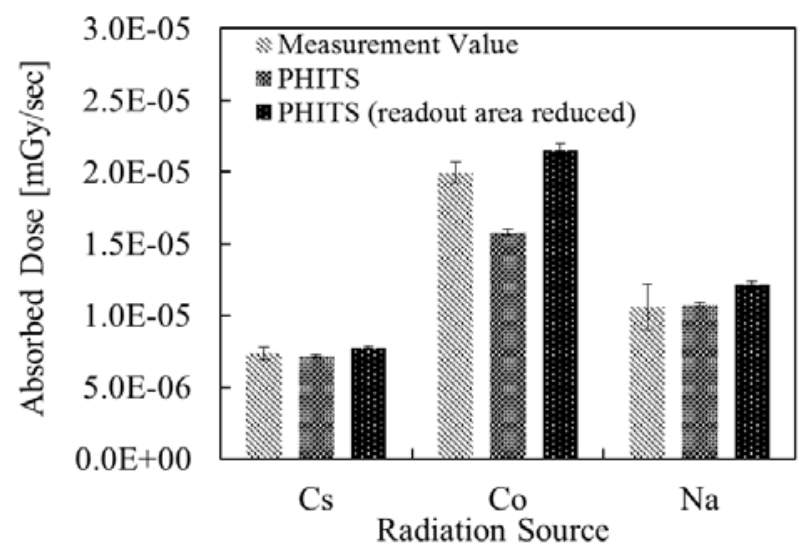

Fig 3-10.a. Comparison result of absorbed dose with recalculated value. RPLGDs were covered without a lead filter.

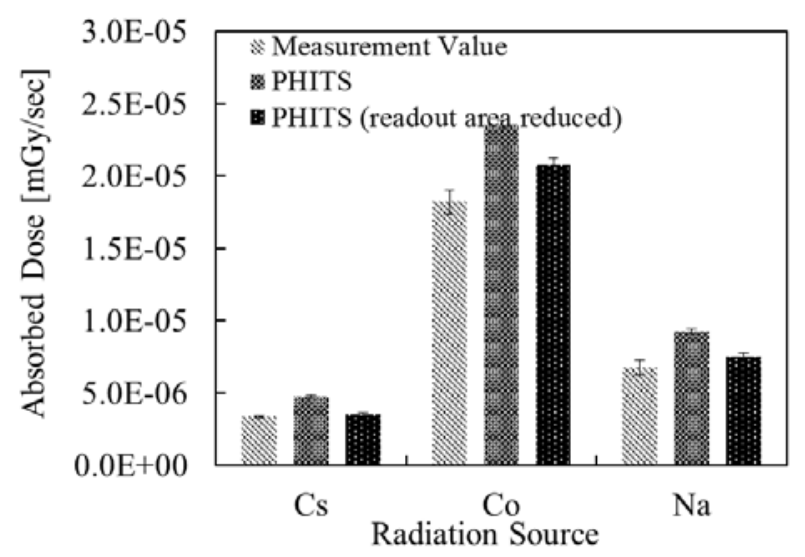

Fig 3-10.b. Comparison result of absorbed dose with recalculated value. RPLGDs were covered with a lead filter.

\section{Conclusion}

In this study, to establish a method of measuring gammaray dose separately at a neutron/gamma-ray mixed field, we proposed "Lead filter Method." As the result of the series experiments in a gamma-ray field, the simulation result especially for the case with a lead filter overestimated the absorbed dose obtained from the measurement. We concluded that the reason of the discrepancy is caused by existence of gradient of the dose distribution in the glass, and the difference of sensitivity to low-energy photons between measurement and theory. 
However, gamma-ray dose could not be reduced sufficiently even if using a lead filter for gamma-rays of which the energy is higher than $1 \mathrm{MeV}$. In the future, to adapt to the actual treatment field of BNCT, we need to re-examine the structure of the lead filter to shield highenergy gamma-rays sufficiently and the size of the glass.

\section{References}

1. Ministry of Health, Labour and Welfare, Japan, Abridged Life table for Japan 2015, http://www.mhlw.go.jp/english/database/dbhw/lifetb15/index.html, (2015)

2. Wolfgang. A.G. Sauerwein, A. Witting, R. Moss, Y. Nakagawa, Neutron Capture, Radiology, Springer, p.1-3 (2012)

3. J.H. Lee, M.S. Lin, S.M. Hsu, I.J. Chen, W.L. Chen, C.F. Wang, Radiation Measurements, 44, 1 (2009)

4. Y. Miyamoto, Y. Takei, H. Nanto, T. Kurobori, A. Konnai, T. Yanagida, A. Yoshikawa, Y. Shimotsuma, M. Sakakura, K. Miura, K. Hirao, Y. Nagashima, T. Yamamoto Radiation Measurements, 46, 12 (2011),

5. Asahi Techno Glass Corporation, "RPL Glass Dosimeter / Small Element System, Dose Ace" (2014)

6. T. Sato, K. Niita, N. Matsuda, S. Hashimoto, Y. Iwamoto, S. Noda, T. Ogawa, H. Iwase, H. Nakashima, T. Fukahori, K. Okumura, T. Kai, S. Chiba, T. Furuta and L. Sihver, J. Nucl. Science Technol. 50, 9 (2013)

7. N. Kadoya,K. Shimomura, S. Kitou, Y. Shiota, Y. Fujita, S. Dobashi, K. Takeda, K. Jingu, H. Matsushita, Y. Namito, S. Ban, S. Koyama, K. Tabushi, Med Phys.,39, 10(2012)

8. M.J. Berger, J.H. Hubbell, S.M. Seltzer, J. Chang, J.S. Coursey, R. Sukumar, D.S. Zucker, and K. Olsen, NIST Standard Reference Database, Xcom; Photon Cross Sections Database, National Institute of Standard Technology (2003) 